\section{$\underset{\substack{\text { hommes } \\ \text { \& migrations }}}{ }$}

\section{Hommes \& migrations}

Revue française de référence sur les dynamiques

migratoires

$1320 \mid 2018$

Au prisme de la consommation

\title{
Les étrangers et les Italiens en Seine-Saint-Denis
}

Un recueil de données par les archivistes du département sur le recensement de 1931

\section{Pierre-Jacques Derainne}

\section{(2) OpenEdition}

\section{Journals}

\section{Édition électronique}

URL : http://journals.openedition.org/hommesmigrations/4070

DOI : 10.4000/hommesmigrations.4070

ISSN : 2262-3353

Éditeur

Musée national de l'histoire de l'immigration

Édition imprimée

Date de publication : 1 janvier 2018

Pagination : $122-125$

ISBN : 978-2-919040-40-7

ISSN : $1142-852 X$

Référence électronique

Pierre-Jacques Derainne, «Les étrangers et les Italiens en Seine-Saint-Denis », Hommes \& migrations [En ligne], 1320 | 2018, mis en ligne le 01 janvier 2018, consulté le 08 janvier 2021. URL : http:// journals.openedition.org/hommesmigrations/4070; DOI : https://doi.org/ERREUR PDO dans / localdata/www-bin/Core/Core/Db/Db.class.php L.34 : SQLSTATE[HY000] [2006] MySQL server has gone away 


\section{LES ÉTRANGERS ET LES ITALIENS EN SEINE-SAINT-DENIS}

\section{UN RECUEIL DE DONNÉES PAR LES ARCHIVISTES DU DÉPARTEMENT SUR LE RECENSEMENT DE 1931}

Par PIERRE-JACQUES DERAINNE.

En l'im 2016, le Musée national de l'histoire de l'immigration, les Archives départementales de la Seine-Saint-Denis et plusieurs services d'archives municipales de ce département ont entrepris de réaliser un recueil de données sur les étrangers des communes du 93 à partir du recensement de 1931. Une attention particulière a été portée aux Italiens pour lesquels les données statistiques étaient plus approfondies. Elles devaient faire ressortir les caractéristiques de cette migration à différentes échelles, telles que la ville, le quartier, la rue, en offrant plusieurs angles d'observation : la situation familiale, l'âge, les provenances, l'activité professionnelle, l'employeur.. Cette opération collaborative faisait écho à l'exposition «Ciao Italia » du Musée, inaugurée en mars 2017.

\section{Une opération collaborative}

Coordonnée par le Musée national de l'histoire de l'immigration et les Archives départementales de Seine-Saint-Denis, l'opération a permis de couvrir 37 communes sur les 40 du département, notamment les grandes communes d'immigration comme Montreuil, Saint-Denis ou Aubervilliers. La collecte est vaste même si les types de données recueillies diffèrent en fonction des communes. Les données sur l'emploi et les employeurs se limitent ainsi à onze communes'. Pour des communes telles que Neuilly-sur-Marne, Pierrefitte-sur-Seine, Gagny..., les informations sur les aires de provenance sont manquantes. Pour La Courneuve, Gagny ou Aulnay-sous-Bois, le nombre d'étrangers est absent. Pour Dugny, Neuilly-Plaisance ou Noisy-le-Sec en revanche, l'étude ne fournit que le nombre d'étrangers... Ajoutons, pour Clichy-sous-Bois et Drancy, le taux important de lieux d'origine inconnus : 41 et $40 \%$.

\section{Les limites d'une source historique}

On sait que le recensement durant l'entre-deuxguerres ne constitue pas une source historique homogène et fiable. En effet, les agents recenseurs 
n'appliquaient pas de la même façon les directives et, en outre, n'étaient pas tous mus par un souci de la précision, surtout quand ils s'adressaient à des individus parlant mal ou pas du tout le français. Tous les individus recensés étaient en outre loin de se montrer coopératifs ${ }^{2}$. Les différences de rédaction sont dès lors manifestes entre les registres de recensement, voire parfois à l'intérieur d'un même registre.

Les erreurs orthographiques des agents, notamment en ce qui concerne les lieux de naissance, sont nombreuses, du fait souvent d'une écriture phonétique, de même que leurs approximations et leurs simplifications relatives à la commune de naissance, au nom ou à la localisation de l'employeur, ou encore à l'activité professionnelle. Il leur arrive ainsi fréquemment de n'indiquer, par exemple, que le pays de naissance d'un individu étranger en omettant la localité ou bien d'inscrire la localité de l'employeur sans préciser le nom de l'entreprise. De plus, on constate en croisant les données de l'état-civil et du recensement, que certaines informations concernant la date ou le lieu de naissance d'un enfant sont erronées du fait d'une fausse déclaration ou d'une erreur de transcription.

Le croisement de sources permet souvent de relativiser l'activité professionnelle inscrite dans le registre. On découvre par l'état civil, par exemple, qu'un Italien répertorié comme "verrier » chez Legras dans le recensement de Pantin de 1921 a silIonné auparavant le nord de la France avec son épouse comme "musicien ambulant ", l'individu ayant vraisemblablement travaillé temporairement comme manœuvre à la verrerie. Ajoutons la difficulté des agents à déterminer la nationalité des enfants de couples étrangers, suite à la loi de
$1927^{3}$, certains agents préférant parfois laisser des cases vides dans la colonne nationalité appliquée aux enfants...

Cette question de la nationalité des enfants contribue vraisemblablement à expliquer les écarts entre les calculs effectués par les services d'archives et les chiffres mentionnés à la fin des registres de recensement. En ce qui concerne Les Lilas par exemple, le registre de recensement de 1931 conservé aux Archives départementales comporte deux chiffres comptabilisant le nombre d'étrangers : 909 écrits à l'encre et 1336 (rectification au crayon placée sous le chiffre précédent) ; les archives comptabilisent quant à elles 1269 étrangers aux Lilas en 1931.

Certains calculs de l'enquête diffèrent en outre de ceux de chercheurs. C'est, par exemple, le cas des Espagnols et des Italiens de Saint-Denis dont le nombre en 1931 s'élève respectivement à 2845 et 1774 selon cette étude et à 3423 et 2083 selon Natacha Lillo ${ }^{4}$.

\section{La contribution associative}

La participation de la Société d'étude historique de Tremblay-en-France en la personne de son président montre l'apport que peuvent fournir les associations à ce type de projet. La Société a offert des informations sur l'implantation italienne à Tremblay en 1931, sur l'entreprise locale familiale La perle de Venise fondée par Vittorio Moretti né à Murano, ainsi que sur les formes d'insertion des immigrés italiens, y compris à travers l'engagement politique: antifascistes partis dans les Brigades internationales, résistants sous I'Occupation.

2. Sur la critique des recensements comme source historique, voir Philippe Videlier, "Problème de fiabilité des recensements : l'exemple de Vénissieux entre les deux guerres ", in Population, vol. 38, n 3, 1983, pp. 598-605. Éric Guichard, Gérard Noiriel (dir),

Construction des nationalités et immigration dans la France contemporaine, Paris, Presses de l'École normale supérieure, 1997.

3. Ce qu'avait déjà remarqué Marie-Claude Blanc-Chaléard dans sa thèse : "D’autres échappent aux statistiques, en particulier les enfants nés après 1927 et que l'on place de facto dans le camp démographique français, selon l'esprit (plus que la lettre) de la loi de naturalisation ". Voir Les Italiens dans l'Est parisien des années 1880 aux années 1960, une histoire d'intégration, Paris, IEP Paris, 1995, p. 206. 4. Natacha Lillo, Histoire des immigrations en île-de-France de 1830 à nos jours, Paris, Publibook, 2012, pp. $58,65$. 


\section{INITIATIVES}

\section{L'apport des cartes et des diagrammes}

Ajoutons que l'étude a donné lieu à la production de quelques cartes et diagrammes réalisés par le Service de l'observatoire de la Seine-Saint-Denis permettant, entre autres, de visualiser spatialement la répartition des populations étrangères ou bien les régions de provenance des migrants italiens et leur répartition territoriale en fonction du métier et du genre. On pourra bien sûr comparer les données et les cartes avec la cartographie en ligne de l'Atlas de l'Immigration en France entre les deux guerres réalisé en 1999 à l'École normale supérieure de Paris à partir des recensements de 1931 et de 1936. Il est, cependant, dommage que plusieurs villes, dont Aubervilliers et La Courneuve, n'aient pas pu être intégrées dans les cartes.

Par ailleurs, la définition des sources de données aurait parfois pu être plus précise. C'est le cas des graphiques en camemberts permettant de visualiser, pour chaque commune du département, les principales régions d'origine des migrants en fonction des pourcentages des groupes régionaux au sein de la commune. Dans la mesure où ne sont prises en compte que les cinq premières régions d'origine, le Frioul, par exemple, est exclu visuellement d'une ville comme Montreuil alors que cette commune apparait, par ailleurs, comme la plus peuplée de Frioulans (en nombre absolu) parmi toutes celles de l'actuelle Seine-Saint-Denis; l'implantation frioulane débordant sur les villes limitrophes de Bagnolet et Romainville.

\section{Une appréhension de l'immigration à l'échelle d'un département}

II n'en demeure pas moins que ce recueil de données offre un ensemble statistique précieux. En effet, il n'existe pas, pour la période de l'entre-deux- guerres et pour l'espace de la banlieue parisienne, de relevé statistique sur l'immigration en général et sur l'immigration italienne en particulier ayant pour base les communes d'une aire géographique aussi importante. On dispose de recherches limitées à un seul quartier parisien (la Villette étudiée par Judith Rainhorn et Kyung II Lee), de monographies communales sur les Italiens à Aubervilliers ou à Noisy-le-Grand..., de travaux d'ampleur à l'échelle de plusieurs communes et quartiers parisiens (Est parisien, Montreuil, Nogent-sur-Marne étudiés par Marie-Claude Blanc-Chaléard). Seul Laurent Couder a choisi d'analyser dans sa thèse les immigrés italiens à l'échelle de la région parisienne durant les années 1920, mais en se servant du recensement de 1926 dont les données sur les étrangers dans le département de la Seine (répartition par villes et par nationalités) ont été publiées dans l'Annuaire statistique de la ville de Paris5.

Ce recueil de données établi à partir du recensement de 1931 offre donc de prometteuses comparaisons avec les données de 1926 pour mieux connaître l'évolution des flux au cours des années 1920 dans la banlieue nord-est de Paris. II permet d'appréhender plus finement l'histoire des migrations au niveau territorial, mais aussi l'histoire sociale de l'urbanisation de la banlieue, puisqu'il concerne aussi bien les communes densifiées de la petite ceinture que celles plus éloignées de la capitale, accueillant de vastes lotissements, telles Drancy, Tremblay, etc.

\section{Une logique migratoire de dispersion}

Deux villes du département, Montreuil et Aubervilliers, se distinguent par l'importance de leur population italienne qui avoisine dans chacune d'entre elles les 3800 individus. Mais, globalement, l'étude souligne la dissémination 
territoriale des immigrés italiens qui s'accentue entre 1926 et 1931, touchant quasiment toutes les villes de l'actuelle Seine-Saint-Denis. Ce phénomène s'effectue dans un contexte de crise du logement, de vieillissement et de saturation des quartiers périphériques de la capitale, souvent composés d'appartements trop exigus pour les familles nombreuses. Cette saturation urbaine s'étend même à certaines villes de la petite couronne telles que Saint-Ouen, Pantin, le Pré-SaintGervais, expliquant l'accroissement limité de l'immigration italienne dans ces communes.

Dès lors, le "quartier italien", marqué par la communautarisation, une relative extension géographique et l'essor de fonctionnalités urbaines complémentaires, s'efface bien souvent au profit du micro quartier, à l'échelle d'un chemin, d'une fraction de rue ou d'une cour. Cette dissémination atténue la visibilité de l'implantation italienne dans l'espace banlieusard et opacifie la notion même de « petite Italie ".

\section{Étendre l'enquête et comparer les territoires}

Bien entendu, si l'échelle du département a du sens du point de vue de la méthodologie du recueil de données, elle en a moins du point de vue de la géographie historique de l'implantation de l'immigration en banlieue. Les mouvements migratoires n'ont pas épousé les espaces administratifs et il est donc utile de croiser ces données avec celles déjà effectuée dans des départements limitrophes, par exemple le recueil de chiffres effectué en 2012 par la Mission mémoires et identités en Val-de-France sur l'immigration dans les communes de Sarcelles, Garges, Villiersle-Bel, Arnouville ${ }^{6}$ et d'étendre même la collecte à d'autres départements, par exemple le Val-de-Marne.

Au sein de la Seine-Saint-Denis, il faudrait achever l'enquête notamment en ce qui concerne Aubervilliers. Plus généralement, les logiques de mobilité interne qui ont commencé à être mises en lumière entre la Villette, Pantin, Drancy et La Courneuve pourraient être approfondies. II serait en outre pertinent de comparer des populations italiennes au sein de territoires industriels différents : notamment les villes de petite industrie telles que Montreuil ou Bagnolet; les villes d'industries métallurgiques ou chimiques telles qu'Aubervilliers, La Courneuve, Saint-Denis ; les villes du plâtre telle que Gagny ou Livry-Gargan. Des bases de données pourraient être constituées pour faciliter les recherches postérieures, par exemple sur les employeurs d'ouvriers italiens. Afin de mener ces actions, la constitution d'un nouveau groupe de recherche élargi aux archivistes, aux chercheurs, aux associations et au Musée national de l'histoire de l'immigration s'avère déterminante. 